\title{
Comparison of statin plus ezetimibe with double-dose statin on lipid profiles and inflammation markers
}

Na-Qiong Wu, Yuan-Lin Guo, Cheng-Gang Zhu, Ying Gao, Xi Zhao, Di Sun, Jing Sun, Rui-Xia Xu, Geng Liu, Qian Dong and Jian-Jun Li

\begin{abstract}
Background: Achievement of low-density lipoprotein cholesterol (LDL-C) goal is the most important for the patients with atherosclerotic cardiovascular diseases (ASCVD) who received lipid-lowering therapy. It is unclear that whether combination of ezetimibe with statin is superior to double-dose of statin regarding both of the lipid-lowering efficacy and improvement of inflammation in Chinese patients with ASCVD. Therefore, this study was performed to compare the effects of these two regimes on lipid profiles and inflammation markers.

Methods: In this randomized control study, ninety eight patients with ASCVD, who were naïve to statins or other lipidlowering agents, were enrolled into the study, and randomly assigned into two groups, A40 group (atorvastatin $40 \mathrm{mg} /$ $\mathrm{d}, n=50$ ), A20E10 group (atorvastatin $20 \mathrm{mg} / \mathrm{d}$ combined with ezetimibe $10 \mathrm{mg} / \mathrm{d}, n=48$ ). The patients were followed up at week 4 and week 12 after treatment. The lipid profiles and oxidative low-density lipoprotein cholesterol (ox-LDL) were measured at the end of study.

Results: There were no differences in clinical characteristics including lipid, ox-LDL and hypersensitive $C$ reactive protein (Hs-CRP) among groups at baseline. However, the average level of LDL-C was lower in group A20E10 than that in group A40 significantly $(1.59 \pm 0.44 \mathrm{mmol} / \mathrm{L}$ vs $1.99 \pm 0.56 \mathrm{mmol} / \mathrm{L}, p=0.001)$ during follow-up at week 12 after treatment. Importantly, the higher rate of achievement of LDL-C goal was attained at group of combination statin with ezetimibe (79.2\% in group A20E10 vs 50.0\% in group A40, $p=0.016)$. The difference of the level of ox-LDL between both the groups after 12 weeks treatment had not statistical significance (3.63 $\pm 1.13 \mathrm{U} / \mathrm{L}$ in group A20E10 vs $4.14 \pm 1.32 \mathrm{U} / \mathrm{L}$ in group A40, $p=0.077)$.Similarly, the level of Hs-CRP between both the groups after treatment was not significantly different $(p>0.05)$.

Conclusions: In this randomized study, the data showed that a combination of moderate statin and ezetimibe achieved
\end{abstract} more reduction of $L D L-C$ compared to the double-dose statin but similar impact on inflammation markers.

Keywords: Statin, Ezetimibe, Combination, LDL-C, Ox-LDL, Chinese

\section{Introduction}

According to the 2013 American Cardiology College/ American Heart Association (ACC/AHA) guideline of cholesterol management, the patients at very high risk of atherosclerotic cardiovascular diseases (ASCVD) should take high intensity statins whatever the low-density

\footnotetext{
* Correspondence: lijianjun938@126.com

Division of Dyslipidemia, State Key Laboratory of Cardiovascular Disease, Fu Wai Hospital, National Center for Cardiovascular Disease, Chinese Academy of Medical Sciences and Peking Union Medical College, No 167 BeiLiShi Road, XiCheng District, Beijing 100037, China
}

lipoprotein cholesterol (LDL-C) level when initiation statin treatment [1], however, evidences showed that there was racial difference in statin tolerance and safety problems between Chinese and Caucasians [2]. High intensity statins were defined as atorvastatin $40-80 \mathrm{mg} / \mathrm{d}$ or rosuvastatin $20-40 \mathrm{mg} / \mathrm{d}$ in $2013 \mathrm{ACC} / \mathrm{AHA}$ guideline. Unfortunately, in China, rosuvastatin $40 \mathrm{mg} / \mathrm{d}$ is prohibited to prescribe for patients due to no approval from Chinese State Food and Drug Administration (SFDA). In addition, lack of evidence about benefit and safety of atorvastatin $80 \mathrm{mg} / \mathrm{d}$ on Chinese patients supported

(c) The Author(s). 2018 Open Access This article is distributed under the terms of the Creative Commons Attribution 4.0 International License (http://creativecommons.org/licenses/by/4.0/), which permits unrestricted use, distribution, and 
Chinese doctors to prescribe it. Hence, the Chinese adult lipid management guideline (2016 revision edition) do not recommend high-dose statin such as atorvastatin 80 $\mathrm{mg} / \mathrm{d}$ or rosuvastatin $40 \mathrm{mg} / \mathrm{d}$ to use in real world clinical practice [3].

Nevertheless, both 2016 European Society of Cardiology/ European Atherosclerotic Society (ESC/EAS) guideline and 2016 Chinese guideline of cholesterol management recommended that for the patients at very high risk of ASCVD, the goal of LDL-C should be $<1.8 \mathrm{mmol} / \mathrm{L}$ or a reduction of at least $50 \%$ if the baseline level of LDL-C was 1.8-3.5 $\mathrm{mmol} / \mathrm{L}[3,4]$. Moreover, IMPROVE-IT trial (Improved Reduction of Outcomes: Vytorin Efficacy International Trial) demonstrated that combination ezetimibe with simvastatin can reduce the level of LDL-C more when compared with simvastatin monotherapy in the patients with acute coronary syndrome and reduce the rate of major adverse cardiovascular events [5], which might suggest that combination ezetimibe with moderate statin may achieve more LDL-C reduction in Chinese high risk patients compared to double-dose statin.

Oxidative stress plays an important role in atherosclerosis and oxidative low-density lipoprotein cholesterol (ox-LDL) has been demonstrated to be a principle lipid-related marker for predicting the severity and outcomes in patients with ASCVD besides LDL-C and non-high-density lipoprotein cholesterol (non-HDL-C) [6]. Additionally, A very large number of studies have indicated that hypersensitive $\mathrm{C}$-reactive protein (Hs-CRP) was a useful inflammatory marker for judging the prognostic outcomes in a variety of cardiovascular diseases $[7,8]$. Interestingly, previous studies showed a powerful impact of statins on oxidative and inflammatory response such oxLDL and Hs-CRP. Based on these evidences and situation in China, we designed this study to compare combination of statin plus ezetimibe and double-dose statin regard to LDL-C reduction and inflammation markers improvement for the Chinese patients at very high risk of ASCVD.

\section{Methods}

\section{Study design and patient population}

The study protocol was reviewed and approved by the Ethics Committee of FuWai Hospital, and informed consent was obtained from all patients. In our randomized, open-label, prospective study, 98 patients naïve to statins or other lipid-lowering agents were enrolled who were diagnosed as ASCVD including coronary artery disease (CAD) or carotid artery disease. The inclusion criteria was as following, 1) the patients with stable angina received coronary angiography showing at least one coronary artery (left anterior descending artery, left circumflex artery, or right coronary artery) stenosis $\geq 50 \%$, or 2 ) the patient received carotid ultrasound showing at least one carotid artery stenosis $\geq 50 \%, 3$ ) no history of statin treatment or use of other drugs known to affect blood lipids within 3 months, 4) age 18-70. Patients with triglycerides (TG) $\geq 500 \mathrm{mg} / \mathrm{dL}(5.6 \mathrm{mmol} / \mathrm{L})$, previous acute coronary syndrome within 1 month, serious heart failure or arrhythmia, infectious disease within 1 month, serious liver or renal dysfunction, autoimmune disease, malignant tumor, pregnancy or lactation, or psychiatric disorders were excluded from the study. In addition, patients with one of the following laboratory values above 3 times the upper limit of normal laboratory range: serum alanine aminotransferase (ALT), serum aspartate transaminase (AST), and creatine phosphokinase above 5 times the upper limit of normal were also excluded.

\section{Clinical and laboratory assessments}

Venous blood samples were obtained in the morning after $12 \mathrm{~h}$ fasting and were blindly assessed with regard to treatment allocation. All laboratory measurements were performed at the laboratory of Biochemistry of FuWai Hospital. Concentrations of total cholesterol (TC), triglycerides (TG) and high-density lipoprotein cholesterol (HDL-C), Apolipoprotein (Apo) A1 and ApoB, as well as lipoprotein a $[\operatorname{Lp}(a)]$ were measured using an automatic biochemistry analyzer (Hitachi 7150, Tokyo, Japan).TC, TG, HDL-C levels were measured using an enzymatic assay. LDL-C was calculated with the Friedewald formula. Apo A1, ApoB, and Lp(a) levels were measured using a turbidimetric immunoassay. Serum creatinine, liver and muscle enzymes were measured using conventional methods. Plasma levels of ox-LDL were measured by a competitive-enzyme-linked immunosorbent assay using a specific murine monoclonal antibody (4E6) according to the instructions provided by the manufacturer.

\section{Statistical analysis}

Data analyses were performed using SPSS 19.0 statistical software. Measurement data are expressed as the means \pm standard deviations (SD); Enumeration data are expressed as absolute frequencies and percentages. For between-group comparisons of basic demographic characteristics and clinical laboratory indicators, analysis of variance (ANOVA) was used for normally distributed measurement data. The paired $\mathrm{t}$ test was used to compare the baseline and post-treatment at week 4 and week 12 in each group. Independent-samples $t$ test was used for comparison between two treatment groups. The chi-square analysis was used to examine the categorical data between treatment groups. All of the tests were conducted at a level of significance of $p=0.05$. 


\section{Results}

\section{Baseline characteristics}

Ninety-eight patients with ASCVD were enrolled and assigned randomly into two groups, including A40 group (A40, $n=50$ ) and A20E10 group (A20E10, $n=48$ ). The baseline characteristics including age, sex had no significant difference between both the groups (all $p>$ 0.05).Similarly, no significant difference of the average baseline level of TC, TG, HDL-C, LDL-C, Lp(a), ApoB ApoA1, and ox-LDL level was found in both the groups (all $p>0.05$ ). The baseline hepatic and renal function was normal and there was no significant difference in the two groups (all $p>0.05$ ). The difference of the baseline fasting blood glucose level and HsCRP had no significance in both the groups (all $p>0.05$, Table 1 ).

\section{Levels of lipid profile and ox-LDL}

During the follow-up period at week 4 after treatment, the average level of $\mathrm{TC}$ was $3.13 \pm 0.97 \mathrm{mmol} / \mathrm{L}$ (group A20E10), $3.50 \pm 0.79 \mathrm{mmol} / \mathrm{L}$ (group A40), respectively

Table 1 Baseline characteristics of the enrolled patients in each group

\begin{tabular}{|c|c|c|c|}
\hline & A20E10 $(n=48)$ & $\mathrm{A} 40(n=50)$ & $P$ value \\
\hline Age(yrs) & $56 \pm 11$ & $57 \pm 8$ & 0.6014 \\
\hline Male/Female & $35 / 13$ & $36 / 14$ & 0.8207 \\
\hline $\mathrm{TC}(\mathrm{mmol} / \mathrm{L})$ & $4.91 \pm 0.95$ & $4.82 \pm 0.73$ & 0.645 \\
\hline $\mathrm{TG}(\mathrm{mmol} / \mathrm{L})$ & $1.89 \pm 0.73$ & $1.74 \pm 0.79$ & 0.332 \\
\hline $\mathrm{HDL}-\mathrm{C}(\mathrm{mmol} / \mathrm{L})$ & $1.07 \pm 0.25$ & $1.14 \pm 0.31$ & 0.195 \\
\hline $\mathrm{LDL}-\mathrm{C}(\mathrm{mmol} / \mathrm{L})$ & $3.31 \pm 0.89$ & $3.20 \pm 0.90$ & 0.540 \\
\hline Non-HDL-C(mmol/L) & $3.91 \pm 0.91$ & $3.56 \pm 0.78$ & 0.077 \\
\hline $\mathrm{Lp}(\mathrm{a})(\mathrm{mg} / \mathrm{L})$ & $222 \pm 215$ & $160 \pm 195$ & 0.140 \\
\hline $\mathrm{ApoB}(\mathrm{mmol} / \mathrm{L})$ & $1.09 \pm 0.29$ & $1.10 \pm 0.26$ & 0.774 \\
\hline Apa A1 (mmol/L) & $1.30 \pm 0.33$ & $1.45 \pm 0.45$ & 0.063 \\
\hline $\mathrm{ALT}(\mathrm{U} / \mathrm{L})$ & $23.44 \pm 15.36$ & $19.90 \pm 10.84$ & 0.190 \\
\hline $\mathrm{AST}(\mathrm{U} / \mathrm{L})$ & $19.89 \pm 5.99$ & $17.80 \pm 6.02$ & 0.089 \\
\hline TBil(umol/L) & $14.26 \pm 4.19$ & $13.96 \pm 4.28$ & 0.722 \\
\hline DBil(umol/L) & $2.30 \pm 0.64$ & $2.34 \pm 0.71$ & 0.748 \\
\hline Cr(umol/L) & $77.69 \pm 15.57$ & $73.36 \pm 14.79$ & 0.163 \\
\hline $\mathrm{BUN}(\mathrm{mmol} / \mathrm{L})$ & $5.05 \pm 1.07$ & $5.28 \pm 1.16$ & 0.327 \\
\hline $\mathrm{CK}(\mathrm{U} / \mathrm{L})$ & $82.13 \pm 35.11$ & $107.60 \pm 117.44$ & 0.157 \\
\hline $\mathrm{FBG}(\mathrm{mmol} / \mathrm{L})$ & $5.34 \pm 1.00$ & $5.76 \pm 1.24$ & 0.075 \\
\hline $\mathrm{HsCRP}(\mathrm{mg} / \mathrm{dL})$ & $1.74 \pm 1.97$ & $2.45 \pm 3.00$ & 0.178 \\
\hline ox-LDL(U/L) & $6.04 \pm 2.00$ & $6.04 \pm 2.00$ & 0.998 \\
\hline
\end{tabular}

Notes: TC Total cholesterol, TG Triglyceride, HDL-C High-density lipoprotein cholesterol, LDL-C Low-density lipoprotein cholesterol, $L p(a)$ Lipoprotein a, $A p o B$ Apolipoprotein B, ApoAl ApolipoproteinAl, ALT Alanine aminotransferase, AST Aspartate Transaminase, TBil Total Bilirubin, DBil Direct Bilirubin, $\mathrm{Cr}$ Creatinine, CK Creatine Kinase, UA Uric Acid, FBG Fasting Blood Glucose, HsCRP High sensitive $C$ - Reactive Protein, $o x-L D L$ oxidation low-density lipoprotein A20E10 indicates the patients who received Atorvastatin $20 \mathrm{mg}$ qn plus Ezetimibe $10 \mathrm{mg}$ qd,A40 indicates the patients who received Atorvastatin $40 \mathrm{mg}$ qn $(p=0.028)$. The average level of LDL-C was $1.68 \pm$ $0.74 \mathrm{moll} / \mathrm{L}$ (group A20E10), $1.89 \pm 0.63 \mathrm{moll} / \mathrm{L}$ (group A40), respectively $(p=0.143)$. The reduction of average level of TG was 32\% in group A20E10 and 20\% in group A40, respectively $(p>0.05)$. The change of HDL-C level had no significant difference compared with that of baseline in the two groups $(p>0.05)$. The reduction of Non-HDL-C level after treatment was significant when compared with that of baseline in both the groups (1.89 $\pm 0.88 \mathrm{mmol} / \mathrm{L}$ vs $3.91 \pm 0.91 \mathrm{mmol} / \mathrm{L}$ in group $\mathrm{A} 20 \mathrm{E} 10$, $2.22 \pm 0.86 \mathrm{mmol} / \mathrm{L}$ vs $3.56 \pm 0.78 \mathrm{mmol} / \mathrm{L}$ in group $\mathrm{A} 40$, $p<0.001$ respectively).

During the follow-up period at week 12 after treatment, the average level of TC was $3.11 \pm 0.54 \mathrm{mmol} / \mathrm{L}$ (group A20E10), $3.62 \pm 0.63 \mathrm{mmol} / \mathrm{L}$ (group A40), ( $p=$ 0.001 ) respectively. The average level of LDL-C was lower in group A20E10 than that in group A40 significantly $(1.59 \pm 0.44 \mathrm{mmol} / \mathrm{L}$ vs $1.99 \pm 0.56 \mathrm{mmol} / \mathrm{L}, p=$ $0.001)$. However, the percentage reduction of average level of TG was 36\% in group A20E10, $22 \%$ in group A40, respectively $(p>0.05)$, and the change of HDL-C level had no significant difference in the two groups $(p>$ 0.05). The reduction of Non-HDL-C level after treatment was significant when compared with that of baseline in both the groups $(1.89 \pm 0.63 \mathrm{mmol} / \mathrm{L}$ vs $3.91 \pm$ $0.91 \mathrm{mmol} / \mathrm{L}$ in group A20E10, $1.82 \pm 1.18 \mathrm{mmol} / \mathrm{L}$ vs $3.56 \pm 0.78 \mathrm{mmol} / \mathrm{L}$ in group $\mathrm{A} 40, p<0.001$ respectively, Fig. 1a-e). In addition, although ox-LDL level of each group was reduced significantly during follow-up at week 12 when compared with that at baseline (3.63 \pm $1.13 \mathrm{U} / \mathrm{L}$ vs. $6.04 \pm 2.00 \mathrm{U} / \mathrm{L}$ in group A20E10, $4.14 \pm$ $1.32 \mathrm{U} / \mathrm{L}$ vs $6.04 \pm 2.00 \mathrm{U} / \mathrm{L}$ in group $\mathrm{A} 40, p<0.001$, respectively), no significant difference of ox-LDL level after treatment for 12 weeks was found between group A20E10 and group A40 ( $p=0.077$, Fig. 1f).

The higher percentage of achievement of LDL-C goal $(<1.8 \mathrm{mmol} / \mathrm{L})$ was found in group A20E10 compared with that in group $\mathrm{A} 40((79.2 \%$ in group A20E10 vs $50.0 \%$ in group A40, $p=0.016$, Fig. 2).

\section{Hs-CRP}

During the follow-up period at week 4 after treatment, serum Hs-CRP level was $1.41 \pm 2.07 \mathrm{mg} / \mathrm{dl}$ in group A20E10 ( $p=0.772$, when compared with that of baseline), $1.74 \pm 1.66 \mathrm{mg} / \mathrm{dl}$ in group A40, ( $p=0.151$ compared with that of baseline). During the follow-up period at week 12 after treatment, serum Hs-CRP level was $1.65 \pm 2.36 \mathrm{mg}$ / $\mathrm{dl}$ in group A20E10 ( $p=0.850$, when compared with that of baseline), $2.07 \pm 2.56 \mathrm{mg} / \mathrm{dl}$ in group A40, $(p=0.570$ compared with that of baseline). The level of Hs-CRP in the two groups exhibited a tendency of reduction, even though no statistical significance was found when compared with the baseline level (Fig. 3). 


\section{A}

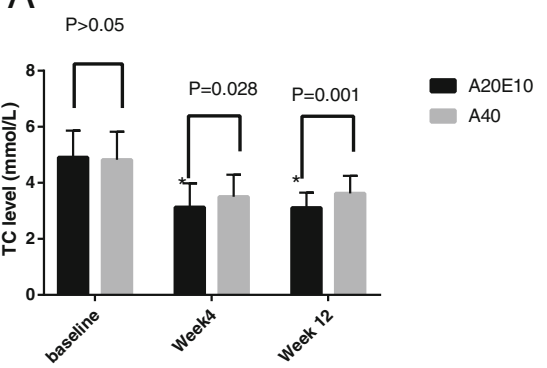

C

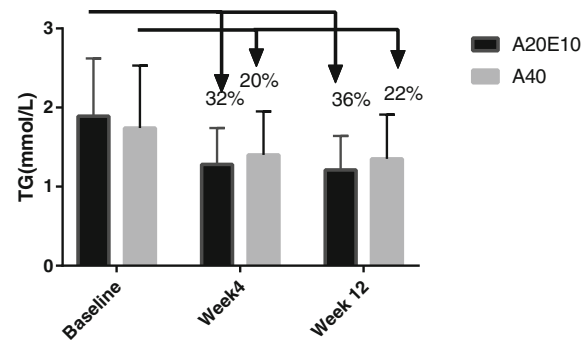

E

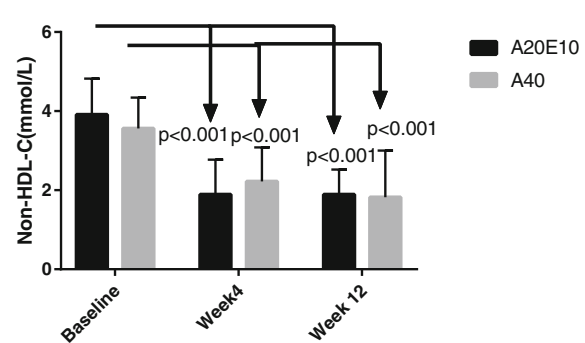

B

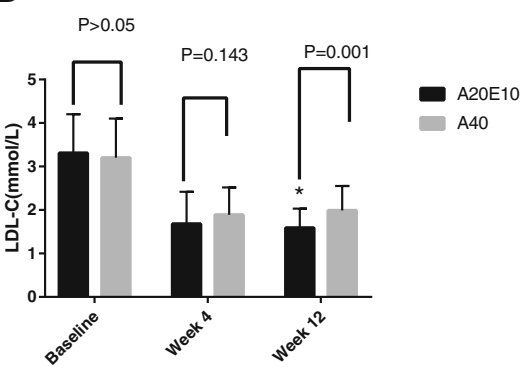

D

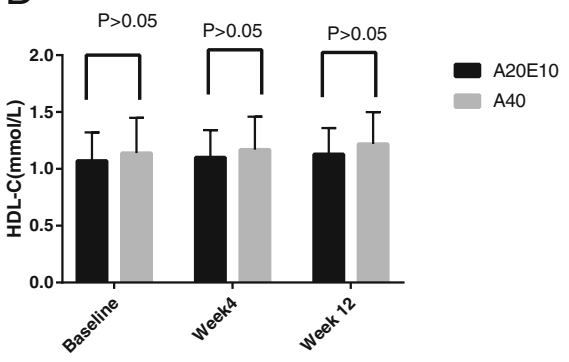

F $\quad P<0.001$

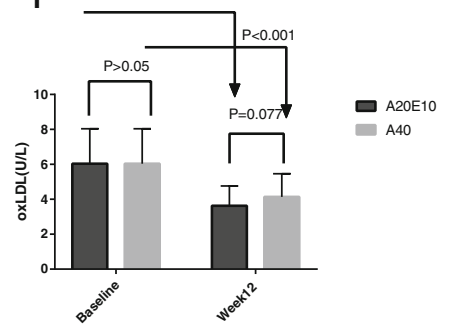

Fig. 1 The change of serum lipid profile levels and ox-LDL in both groups during follow-up. The patients with ASCVD divided into two groups, the combination group who received Ezetimibe $10 \mathrm{mg} / \mathrm{d}$ with atorvastatin $20 \mathrm{mg} / \mathrm{d}$ (group A20E10, $n=48$ )and statin alone group who received atorvastatin $40 \mathrm{mg} / \mathrm{d}$ (group A40, $n=50$ ).Serum levels of lipid profile were measured at baseline, week 4 and week 12 after treatment. a average TC levels, ${ }^{*}$ Indicate the level of TC in group A20E10 was lower than that in group A40 significantly at week 4 and week 12 after treatment. $\mathbf{b}$ average LDL-C level, Indicate the level of LDL-C in group A20E10 was lower than that in group A40 significantly at week 12 after treatment. $\mathbf{c}$ average TG level. $\mathbf{d}$ average HDL-C level. e average Non-HDL-C level. $\mathbf{f}$ average ox-LDL level. Notes: A20E10 indicates the patients who received Atorvastatin $20 \mathrm{mg}$ qn plus Ezetimibe $10 \mathrm{mg}$ qd,A40 indicates the patients who received Atorvastatin $40 \mathrm{mg}$ qn

\section{Safety evaluation}

During the follow-up period at week 12 after treatment, all the patients $(n=98)$ were informed to be followed up. Self-reported side effects including fatigue or muscle pain and memory decline were recorded, and no other complains about side effects were reported. The number of the patients who complained fatigue or muscle pain was as followings, $1(2.0 \%)$ in group A40, 0 in group A20E10. The number of the patients who had ALT or AST elevation to 1-3 times of ULN was as followings, $2(4.2 \%)$ in group A20E10, 3(6.0\%) in group A40. One patient (2.0\%) in group A40 but nobody in group A20E10 had CK elevation 1-5times of ULN. No patients had ALT or AST elevation $>3$ times of ULN or CK elevation $>5$ times of ULN in both the groups.
No significant differences in ALT, AST and CK were observed in both the groups during the follow-up periods both at week 4 and week 12 after treatment (all $p>0.05$, Table 2).

\section{Discussion}

In this randomized study, we found that atorvastatin 20 $\mathrm{mg} / \mathrm{d}$ plus ezetimibe $10 \mathrm{mg} / \mathrm{d}$ for 12 weeks therapy achieved more TC, LDL-C reduction for the Chinese patients with very high risk of cardiovascular diseases. Besides, the similar impact of different intensive lipidlowering patterns on ox-LDL and Hs-CRP was found. Thereby, the study may provide additional or novel information regarding the clinical implication of statin plus ezetimibe lipid-lowering strategy. 


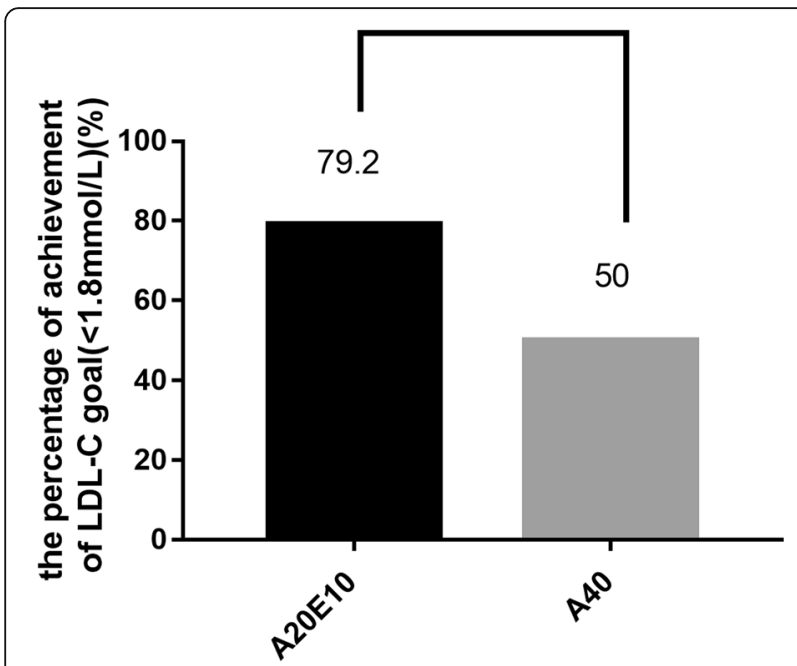

Fig. 2 The comparison of the pecentage of achievement of $L D L-C$ goal $(<1.8 \mathrm{mmol} / \mathrm{L})$ between the group A2OE10 and the group A40. Notes: A20E10 indicates the patients who received Atorvastatin 20 $\mathrm{mg}$ qn plus Ezetimibe $10 \mathrm{mg}$ qd A40 indicates the patients who received Atorvastatin $40 \mathrm{mg}$ qn

Firstly, we investigated the efficacy of two lipid-lowering regimes in enrolled patients. As presented in Fig. 1, the lipid-lowering therapies such as atorvastatin $20 \mathrm{mg} / \mathrm{d}$ plus ezetimibe $10 \mathrm{mg} / \mathrm{d}$ and atorvastatin $40 \mathrm{mg} / \mathrm{d}$ were compared in the aspect of lipid-lowering efficacy. The lipid-lowering efficacy of both strategies was in accordance with the results reported in previous studies [9-11]. Abundant evidences demonstrated that the lower the level of LDL-C reached, the better the reduction of cardiovascular events [12-14]. During the follow-up period at week 12 after treatment, the further reduction of $\mathrm{TC}$ and LDL-C level was found when compared with those in the follow-up period, at week 4 after treatment, suggesting a

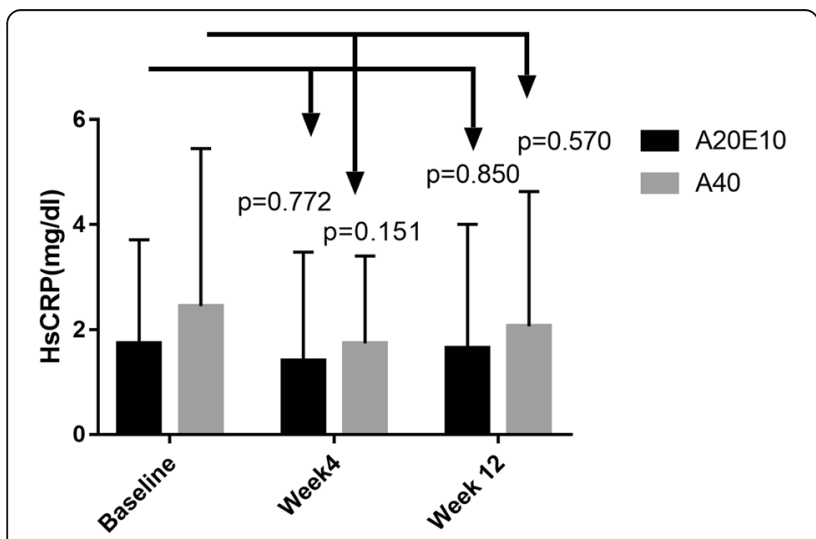

Fig. 3 The comparison of average HsCRP level between the group A20E10 and the group A40 during follow-up. Notes: A20E10 indicates the patients who received Atorvastatin $20 \mathrm{mg}$ qn plus Ezetimibe $10 \mathrm{mg}$ qd A40 indicates the patients who received Atorvastatin $40 \mathrm{mg}$ qn
Table 2 Safety markers at baseline, week 4 and week 12 by group

\begin{tabular}{cccc}
\hline & A20E10 $(n=48)$ & A40 $(n=50)$ & $P$ value \\
\hline ALT(U/L) & & & \\
Baseline & $23.44 \pm 15.36$ & $19.90 \pm 10.84$ & 0.190 \\
Week 4 & $30.20 \pm 14.52$ & $27.51 \pm 16.78$ & 0.410 \\
Week 12 & $31.39 \pm 19.38$ & $28.39 \pm 22.36$ & 0.552 \\
AST(U/L) & & & \\
Baseline & $19.89 \pm 5.99$ & $17.80 \pm 6.01$ & 0.089 \\
Week 4 & $22.84 \pm 5.69$ & $21.24 \pm 6.92$ & 0.231 \\
Week 12 & $31.39 \pm 19.38$ & $28.39 \pm 22.36$ & 0.321 \\
CK(U/L) & & & \\
Baseline & $82.13 \pm 35.11$ & $107.60 \pm 117.44$ & 0.157 \\
Week 4 & $95.05 \pm 35.58$ & $100.86 \pm 40.46$ & 0.466 \\
Week 12 & $104.39 \pm 48.86$ & $115.60 \pm 57.01$ & 0.386 \\
\hline
\end{tabular}

Notes: A20E10 indicates the patients who received Atorvastatin $20 \mathrm{mg}$ qn plus Ezetimibe $10 \mathrm{mg}$ qd,A40 indicates the patients who received Atorvastatin $40 \mathrm{mg}$ qn

stable lowering LDL-C effect by both moderate dose statin plus ezetimibe and double-dose statin.

Another important concern with regard to lipid-lowering therapy is safety. In the current study, we also evaluated side effects of the different lipid-lowering strategies although the sample size of the study is small. Data showed that no serious side effects happened in both groups. However, only occasional mild hepatic dysfunction or mild CK elevation was found in both the groups through more detailed observations. Thus, the combination of ezetimibe with atorvastatin had good tolerance and safety of lipid-lowering strategy.

In addition, recent reports have suggested that a synergistic effect can be obtained by concomitant administration of the cholesterol absorption inhibitor ezetimibe and a statin [15-19]. Torimoto $\mathrm{K}$ et al. performed the study to examine which was more effective to add ezetimibe or to increase the statin dose on LDL-C and lipoproteins in patients with type 2 diabetes who were already being treated with satins. Interestingly, they found that compared with increasing the dose of rosuvastatin, the combination of rosuvastatin and ezetimibe not only achieves quantitative but also qualitative improvement of serum lipid levels in type 2 diabetic patients, suggesting that this combination can suppress the progression of atherosclerosis [15].

Oxidation stress and inflammation represent integral features of atherogenesis and vascular diseases. It has been reported that ox-LDL is reliable marker for predicting the prognosis in ASCVD. In the present study, therefore, we investigated the change of ox-LDL level after 12 weeks lipid-lowering treatment, the level of ox-LDL was reduced significantly in both groups including combination of ezetimibe with atorvastatin (A20E10) and double-dose atorvastatin (A40). Interestingly, we 
found that in the two groups of our study, ox-LDL level decreased significantly and was in parallel with the reduction of LDL-C level, which is consistent with previous studies. Similar effect of ox-LDL reduction was found in both combination treatment group and doubledose statin group. Aydin MU et al. compared the effects of $80 \mathrm{mg}$ daily dose of atorvastatin and $20 \mathrm{mg}$ daily dose of rosuvastatin on lipid profiles and the levels of ox-LDL and inflammatory markers in ST elevation myocardial infarction (STEMI), and they found that both statin treatment regimes have comparable effects on LDL-C, ox-LDL and inflammatory markers [20]. However, in a prospective, open-label trial which enrolled 260 patients with coronary artery disease, assigned randomly into simvastatin mono-therapy group or simvastatin plus ezetimibe group, the results showed co-administration of ezetimibe with statins further lowered LDL-C levels $(83 \pm 23$ $\mathrm{mg} / \mathrm{dl}$ in $\mathrm{S}$ versus $67 \pm 23 \mathrm{mg} / \mathrm{dl}$ in $\mathrm{E}+\mathrm{S}$; $p<0.0001$ ), with significant decreases in ox-LDL level [21].

As we well known, Hs-CRP is an important inflammatory marker of ASCVD and adds prognostic information on cardiovascular risk comparable to blood pressure or cholesterol. In current U.S. guidelines, Hs-CRP carries a class IIb assessment and is most appropriate in primary prevention when clinical decisions to initiate statin therapy are uncertain. Ongoing multinational trials are pursuing whether reducing inflammation will decrease vascular event rates [22]. Further, randomized trial data addressing Hs-CRP have been central to understanding the anti-inflammatory effects of statin therapy and have consistently demonstrated on-treatment Hs-CRP levels to be as powerful a predictor of residual cardiovascular risk as on-treatment levels of LDL-C [23]. In the small sample size study with 69 patients who were allocated into simvastatin alone or simvastatin combined with ezetimibe group randomly, Krysiak $\mathrm{R}$ reported that the combination therapy was superior to simvastatin in influencing plasma level of Hs-CRP [24]. In the present study, the level of Hs-CRP exhibited a tendency of reduction in both the groups, when compared with the baseline level. Our data was similar to IMPROVE-IT study with regard to the changes of Hs-CRP following lipid-lowering treatment and suggested that reduction of Hs-CRP mainly depended on whether a statin is used [5].

Limitations of the current study include the open-label design, the absence of a placebo group and the relatively short duration. In addition, the small sample size is the other limitation of the study, more study is needed to confirm our findings in large sample size.

In conclusion, in this randomized study, the data showed that a combination of moderate statin and ezetimibe achieved more reduction of LDL-C compared to the double-dose statin but similar impact on inflammation markers.

\section{Abbreviations}

ACC/AHA: American Cardiology College/American Heart Association; ALT: Alanine aminotransferase; ANOVA: Analysis of variance; Apo A1: ApolipoproteinsA1; ASCVD: Atherosclerotic cardiovascular diseases; AST: Aspartate transaminase; CAD: Coronary artery disease; ESC/EAS: European Society of Cardiology/European Atherosclerotic Society; HDL-C: high-density lipoprotein cholesterol; HsCRP: Highsensible C reactive protein; IMPROVE-IT: Improved Reduction of Outcomes: Vytorin Efficacy International; LDL-C: low-density lipoprotein-cholesterol; Lp(a): lipoprotein a; non-HDL-C: Non-high-density lipoprotein cholesterol; ox-LDL: Oxidative lowdensity lipoprotein cholesterol; SD: Standard deviations; SFDA: State Food and Drug Administration; STEMI: ST elevation myocardial infarction; TC: Total cholesterol; TG: Triglycerides

\section{Acknowledgments}

The authors appreciate the financial support of this study by the Captial Health Development Fund and CAMS Major Collaborative Innovation Project.

\section{Funding}

This work was partially supported by the Capital Health Development Fund (201614035) and CAMS Major Collaborative Innovation Project (2016-12M-1- 011) awarded to Dr. Jian-Jun Li, MD, PhD.

Availability of data and materials

Please contact author for data requests.

\section{Authors' contributions}

$J \mathrm{JL}$ and NQW were in charge of the design of this study; NQW, XZ, DS, JS, RXX, GL and QD carried out the experiments; NQW, YLG, CGZ, YG mainly analyzed the experimental data and results. NQW and JJL wrote the manuscript. All authors read and approved the final manuscript.

\section{Ethics approval and consent to participants}

Our study complied with the Declaration of Helsinki and was approved by the hospital's ethical review board (Fu Wai Hospital \& National Center for Cardiovascular Diseases, Beijing, China). Informed written consents were obtained from all the participants.

Consent for publication

Not applicable

Competing interests

The authors declare that they have no competing interests.

\section{Publisher's Note}

Springer Nature remains neutral with regard to jurisdictional claims in published maps and institutional affiliations.

Received: 28 May 2018 Accepted: 12 November 2018

Published online: 23 November 2018

\section{References}

1. Stone NJ, Robinson JG, Lichtenstein AH, Bairey Merz CN, Blum CB, Eckel RH, et al. 2013 ACC/AHA guideline on the treatment of blood cholesterol to reduce atherosclerotic cardiovascular risk in adults: a report of the American College of Cardiology/American Heart Association task force on practice guidelines. Circulation. 2014;129(25 Suppl 2):S1-S45.

2. HPS2-THRIVE Collaborative Group, Landray MJ, Haynes R, Hopewell JC, Parish S, Aung T, et al. Effects of extended-release niacin with laropiprant in high-risk patients. N Engl J Med. 2014;371:203-12.

3. Catapano AL, Graham I, De Backer G, Wiklund O, Chapman MJ, Drexel H, et al. 2016 ESC/EAS guidelines for the Management of Dyslipidaemias. Eur Heart J. 2016:37:2999-3058.

4. Joint Committee on guidelines for the prevention and control of dyslipidemia in Chinese adults. Chinese adult dyslipidemia guideline (2016 revision edition). Chin Cir J. 2016:31:937-53.

5. Cannon CP, Blazing MA, Giugliano RP, McCagg A, White JA, Theroux P, et al. Ezetimibe added to statin therapy after acute coronary syndromes. N Engl J Med. 2015;372:2387-97.

6. Kei A, Tellis C, Liberopoulos E, Tselepis A, Elisaf M. Effect of switch to the highest dose of rosuvastatin versus add-on-statin fenofibrate versus add-on- 
statin nicotinic acid/laropiprant on oxidative stress markers in patients with mixed dyslipidemia. Cardiovasc Ther. 2014;32:139-46.

7. Serban MC, Banach M, Mikhailidis DP. Clinical implications of the IMPROVEIT trial in the light of current and future lipid-lowering treatment options. Expert Opin Pharmacother. 2016;17:369-80.

8. Zhang Y, Li S, Guo YL, Wu NQ, Zhu CG, Gao Y, et al. Is monocyte to HDL ratio superior to monocyte count in predicting the cardiovascular outcomes: evidence from a large cohort of Chinese patients undergoing coronary angiography. Ann Med. 2016;48:305-12.

9. Takase S, Matoba T, Nakashiro S, Mukai Y, Inoue S, Oi K, et al. Ezetimibe in combination with statins ameliorates endothelial dysfunction in coronary arteries after stenting: the CuVIC trial (effect of cholesterol absorption inhibitor usage on target vessel dysfunction after coronary stenting), a Multicenter Randomized Controlled Trial. Arterioscler Thromb Vasc Biol. 2017:37:350-8

10. Homma K, Homma Y, Ozawa H, Shiina Y, Shibata T, Yoshida T, et al. Comparison of the effects of low-dose rosuvastatin on plasma levels of cholesterol and oxidized low-density lipoprotein in 3 ultracentrifugally separated low-density lipoprotein subfractions. J Clin Lipidol. 2015;9:751-7.

11. Nishikido T, Oyama J, Keida T, Ohira H, Node K. High-dose statin therapy with rosuvastatin reduces small dense LDL and MDA-LDL: the standard versus high-dose therApy with Rosuvastatin for lipiD lowering (SARD) trial. J Cardiol. 2016;67:340-6.

12. Cholesterol Treatment Trialists' (CTT) Collaborators, Mihaylova B, Emberson f, Blackwell L, Keech A, Simes J, et al. The effects of lowering LDL cholesterol with statin therapy in people at low risk of vascular disease: meta-analysis of individual data from 27 randomised trials. Lancet. 2012;380:581-90.

13. Li XL, Li JJ, Guo YL, Zhu CG, Xu RX, Li S, et al. Association of preprocedural low-density lipoprotein cholesterol levels with myocardial injury after elective percutaneous coronary intervention. J Clin Lipidol. 2014;8:423-32.

14. Li XL, Guo YL, Zhu CG, Xu RX, Qing P, Wu NQ, et al. Relationship of highdensity lipoprotein cholesterol with periprocedural myocardial injury following elective percutaneous coronary intervention in patients with low-density lipoprotein cholesterol below 70 mg/dL. J Am Heart Assoc. 2015;4:e001412.

15. Torimoto K, Okada Y, Mori H, Hajime M, Tanaka K, Kurozumi A, et al. Efficacy of combination of ezetimibe $10 \mathrm{mg}$ and rosuvastatin $2.5 \mathrm{mg}$ versus rosuvastatin $5 \mathrm{mg}$ monotherapy for hypercholesterolemia in patients with type 2 diabetes. Lipids Health Dis. 2013;12:137.

16. Tsai NW, Lee LH, Huang CR, Chang WN, Chang YT, Su YJ, et al. Statin therapy reduces oxidized low density lipoprotein level, a risk factor for stroke outcome. Crit Care. 2014;18:R16.

17. Moutzouri E, Liberopoulos EN, Tellis CC, Milionis HJ, Tselepis AD, Elisaf MS. Comparison of the effect of simvastatin versus simvastatin/ezetimibe versus rosuvastatin on markers of inflammation and oxidative stress in subjects with hypercholesterolemia. Atherosclerosis. 2013;231:8-14.

18. Huang B, Cheng Y, Xie Q, Lin G, Wu Y, Feng Y, et al. Effect of $40 \mathrm{mg}$ versus $10 \mathrm{mg}$ of atorvastatin on oxidized low-density lipoprotein, high-sensitivity C-reactive protein, circulating endothelial-derived microparticles, and endothelial progenitor cells in patients with ischemic cardiomyopathy. Clin Cardiol. 2012:35:125-30.

19. Pesaro AE, Serrano CV Jr, Fernandes JL, Cavalcanti AB, Campos AH, Martins HS, et al. Pleiotropic effects of ezetimibe/simvastatin vs. high dose simvastatin. Int J Cardiol. 2012;158:400-4

20. Aydin MU, Aygul N, Altunkeser BB, Unlu A, Taner A. Comparative effects of high-dose atorvastatin versus moderate-dose rosuvastatin on lipid parameters, oxidized-LDL and inflammatory markers in ST elevation myocardial infarction. Atherosclerosis. 2015;239:439-43.

21. Homma Y, Michishita I, Hayashi H, Shigematsu H. Kanagawa lipid research group. Effects of low-dose simvastatin on the distribution of plasma cholesterol and oxidized low-density lipoprotein in three ultra-centrifugally separated low-density lipoprotein subfractions: 12-month, open-label trial. J Atheroscler Thromb. 2010;17:1049-53.

22. Ridker PM. A Test in Context: High-sensitivity C-reactive protein. J Am Coll Cardiol. 2016;67:712-23.

23. Ridker PM. From C-reactive protein to Interleukin-6 to Interleukin-1: moving upstream to identify novel targets for Atheroprotection. Circ Res. 2016;1 18:145-56.

24. Krysiak R, Zmuda W, Okopien B. The effect of simvastatin-ezetimibe combination therapy on adipose tissue hormones and systemic inflammation in patients with isolated hypercholesterolemia. Cardiovasc Ther. 2014;32:40-6.

Ready to submit your research? Choose BMC and benefit from:

- fast, convenient online submission

- thorough peer review by experienced researchers in your field

- rapid publication on acceptance

- support for research data, including large and complex data types

- gold Open Access which fosters wider collaboration and increased citations

- maximum visibility for your research: over $100 \mathrm{M}$ website views per year

At BMC, research is always in progress.

Learn more biomedcentral.com/submissions 